\title{
Supernumerary Kidney with S-shaped Fusion Anomaly: A Rare Congenital Renal Anomaly
}

\author{
Beenish Khan, Chandrashekhara SH \\ Department of Radiodiagnosis, All India Institute of Medical Sciences, Delhi, India.
}

\section{Corresponding Author: \\ Dr Beenish Khan \\ Email: kbeenish540@gmail.com}

This is an Open Access article distributed under the terms of the Creative Commons Attribution License (creativecommons.org/ licenses/by/3.0).

Received Accepted

Published

June 13,2021

July 19, 2021

August 25, 2021

\begin{abstract}
Background: Supernumerary kidneys are rare congenital renal anomaly, most of which are incidentally detected. These are distinguished from duplex kidneys due to presence of separate capsule and separate arterial supply to each kidney. Ultrasonography may detect this rare anomaly but may be insufficient in evaluation of renal vasculature and ureteric drainage due to bowel gases. Cross sectional imaging may better depict morphology of these kidneys and complications like calculi, hydro/pyonephrosis and even renal cancers that are associated with them. Case Report: We report a case having supernumerary kidney along with S-shaped fusion anomaly on left side, incidentally detected in a 50 years old woman. It was smaller in size than the native kidney, had a separate ureter and arterial supply. No calculi/hydronephrosis or malignancy was present in our case. Conclusion: Supernumerary kidney with S-shaped fusion anomaly is a rare congenital renal anomaly, associated with complications like calculi, infections, malignancies. It is also important to distinguish these from other congenital renal anomaly and precisely assess its vascular supply and urinary drainage in renal transplant patients.
\end{abstract}

Keywords: Calculi, Drainage, Kidney, Ultrasonography, Urogenital Abnormalities.

\section{Introduction}

Supernumerary kidneys are rare congenital renal anomalies, most of which are detected incidentally and are associated with other congenital anomalies and complications like urinary stasis, hydronephrosis, calculus and pyonephrosis [1]. A few cases were also associated with renal cancers in the native as well as supernumerary kidneys [2]. With increasing number of transplants in today's time, it is important to understand the imaging characteristics and embryogenesis of this rare entity. These can be incidentally found in donor where it becomes important to differentiate them from crossed fused renal ectopia or bifid collecting system as the management may differ and careful dissection may be required to preserve renal function [3]. We thus describe imaging characteristics of this rarely encountered anomaly which may aid in the early identification of complications and screening of cancers associated with it.

\section{Case Report}

A 51-year-old South-East Asian woman presented to our hospital with an abdominal lump which on subsequent clinical and radiological imaging was suspicious for ovarian carcinoma. She had no other medical history. Her blood pressure was normal. Oophorectomy was done for her in our hospital. Coincidentally, on ultrasound, supernumerary kidney was found on the left side. It was smaller in size than the native kidney, located cranial in relation to the latter and parenchyma was fused with the inferiorly placed native kidney [Fig.1]. CT scan revealed normal contrast opacification of all three kidneys. The renal axis of supernumerary kidney was oriented anteromedially while inferiorly placed native kidney had rotational anomaly 
with its renal pelvis directed anterolaterally. The two kidneys were thus fused in S-shaped manner [Fig.2]. The total number of calyces in supernumerary kidney and the fused native kidney were higher than the contralateral side. There were two separate ureters draining each kidney. The ureter of native malrotated kidney had an oblique course, originating anterolaterally and then coursing medially to lie anterior to Psoas muscle at L3 level. Both kidneys received separate blood supply from aorta, single renal artery supplied the superiorly placed supernumerary kidney while two renal arteries including one accessory renal artery supplied the native kidney. No calculus or hydronephrosis was noted in either kidney. No focal lesion was seen. The right kidney was normally placed in right lumbar region and had a normal shape, single vascular supply and ureter.

Her glomerular filtration rate and kidney function test including blood urea nitrogen and serum creatinine were in normal range. The main differential diagnosis is duplex system, where the ureteric bud divides to form two collecting system in the same metanephros. These can be differentiated from supernumerary kidneys on cross-sectional imaging due to presence of single renal mass, same number of calyces as opposite side, single capsule and a single vascular supply [1]. The imaging features may also closely resemble crossed fused ectopia, where two kidneys are present on one side of abdomen and are fused, often in S-shaped manner. Since, there are two kidneys, two renal masses would be seen, each having its own capsule, vascular supply and ureteric drainage. These can be distinguished from supernumerary kidney due to absence of kidney in contralateral renal fossa. Also, unlike latter, the two renal tissues in crossed fused ectopia are usually of similar size. Careful diagnosis is important especially in cases of transplant where excision of such kidney would render a patient to acute renal insufficiency.
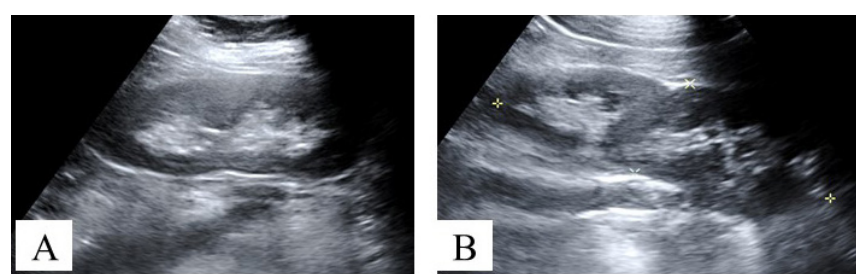

Fig.1: Ultrasound images showing single right kidney and with a smaller supernumerary kidney on left side fused to the parenchyma of caudally placed native kidney.

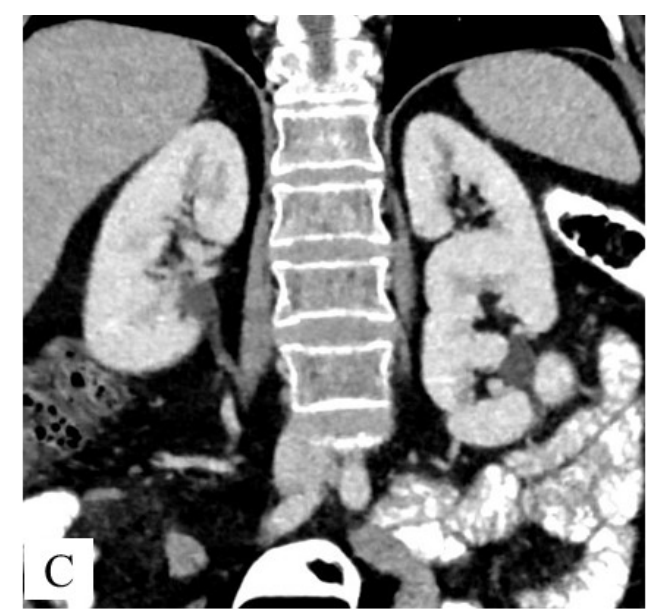

Fig.2: Coronal reformatted CT of supernumerary kidney showing rotation anomaly in inferiorly-placed native kidney with its renal axis directed anterolaterally. The supernumerary kidney shows normally orientation.

\section{Discussion}

Supernumerary kidneys arise when two metanephric blastema arise in a single nephrogenic cord. Hence, these have a separate capsule and separate vascular supply from aorta. The parenchyma may or may not be partially fused. These must not be confused with duplex collecting system where there is a single capsule and a common vascular supply to the two moieties as they arise from a single metanephros however ureteric bud bifurcates to form two excretory systems [4]. Most of them occurs on the left hand with the supernumerary kidney located mostly caudal to the native kidney and in these cases, urinary drainage is mostly into a bifid ureter whereas two separate ureters drain the kidneys when supernumerary kidney is 
located cranial to the native kidney. In the latter case, Weigert Meyer law may be observed [5]. Sometimes the ureter of the supernumerary kidney may be associated with an ectopic opening, such as into the vagina [4].

Supernumerary kidneys are often incidentally diagnosed with mean age of diagnosis 34 years. Abdomen radiograph may reveal enlarged renal shadow which on sonography may show two distinct parenchyma that may be partially fused and have separate pelvi-calyceal system. On intravenous pyelography, when a bifid or double ureter is encountered incidentally the main differential remains duplex collecting system or very rarely supernumerary kidney, the distinction of which may not be possible on pyelography. Multiphasic CT or MRI best depicts anatomy, vascular supply, ureteric drainage, associated complications like obstruction, pyelonephritis, hydronephrosis or renal carcinoma. MRI being radiation free modality may be preferred in children, pregnant women as well patients with kidney disease where a noncontrast MR angiogram may provide sufficient information. Nuclear scintigraphy may be done to assess function of the two kidneys.

Incidental diagnosed supernumerary kidney require no treatment and a follow up can be advised to detect complications [5]. Symptomatic treatment for complications like small calculi may be done. If the kidney is non-functional or harbor malignancy, then nephrectomy is the preferred treatment. There is paucity of literature regarding their encounter and management during transplant however one case has been reported where accessory left renal artery was documented pre-operatively. On vascular anastomosis with the recipient, a non-perfused mass was found at the hilum of the native kidney. This on frozen section turned out to be supernumerary kidney being fused to the hilum of native kidney. It was grossly hydronephrotic and non-functional, and thus resected. Thus, a careful pre-transplant diagnosis may help surgeons to plan dissection and anastomosis and prevent inadvertent resection or mal-perfusion of these kidneys [3]. Follow up to screen complications may be advised. The period and duration of follow up is not described in the literature however it may be advised to image the patient when symptoms develop or 3-5 yearly, whichever is earlier. Symptomatic treatment may be offered for small calculi. Nephrectomy is usually reserved for non-functional kidneys or complications like malignancy [5].

\section{Conclusion}

Supernumerary kidneys are rare congenital renal anomalies where an extra kidney with its own capsule, vascular supply and ureteric drainage is present in the abdomen due to two metanephric blastema being penetrated by ureters. These are smaller in size than native kidneys, often located on left side fused to the native kidney. Complications due to stasis like calculi, hydronephrosis, pyelonephritis and renal cancers can occur and warrants a careful search and screening. Differentiating these from duplex pelvicalyceal system, accessory renal artery or crossed fused ectopia is important, particularly in cases of transplant.

Contributors: BK: manuscript writing, imaging; $\mathrm{CSH}$ : manuscript editing, imaging. BK will act as a study guarantor. Both authors approved the final version of this manuscript and are responsible for all aspects of this study.

Funding: None; Competing interests: None stated.

\section{References}

1. Rehder P, Rehwald R, Böhm JM, Grams AE, Loizides A, Pedrini M, et al. Supernumerary kidneys: a clinical and radiological analysis of nine cases. BMC Urol. 2019;19:93.

2. Gao X, Xing Q, Luo X, Cao T, Zhang S, Yang F, Fan D, et al. Right supernumerary kidney with urothelial carcinoma: A case report. Medicine (Baltimore). 2020;99(38):e22329.

3. Paramesh AS, Volk AS, Zurawin J, Thomas R, Biow S. Management of a donor supernumerary kidney during renal transplantation: A Case Report. Transplantation Case Reports. 2020;1-3.

4. Tada Y, Kokado Y, Hashinaka Y, Kadowaki T, Takasugi Y, Shin T, et al. Free super-numerary kidney: a case report and review. J Urol. 1981:126:231-232.

5. Suresh J, Gnanasekaran N, Dev B. Fused supernumerary kidney. Radiol Case Reports. 2011;6(4):6-9. 